\title{
An analytical solution for one-dimensional contaminant transport in a double-layered contaminated soil with imperfect diffusion boundaries
}

\author{
Xibin $\mathrm{Li}^{1}$, Jianchao Sheng ${ }^{2}$, Zhiqing Zhang ${ }^{{ }^{\dagger}}$, Zhe Wang $^{{ }^{+}}$ \\ ${ }^{1}$ School of Landscape Architecture, Zhejiang A \& F University, Hangzhou, Zhejiang 311300, China \\ ${ }^{2}$ School of Civil Engineering, Shaoxing University, Shaoxing, Zhejiang 312000, China \\ ${ }^{3}$ School of Civil Engineering, Zhejiang University of Technology, Hangzhou, Zhejiang 310000, China
}

\begin{abstract}
An analytical method is proposed to solve the contaminant transport in a double-layered contaminated soil with imperfect diffusion boundaries. By virtue of the separation of variables scheme, the governing equation for contaminant diffusion is split into two ordinary differential equations, and the corresponding general solutions are obtained. By utilizing the initial, continuity and boundary conditions of the system, and the orthogonality of trigonometric functions, the analytical solution for contaminant concentration in the double-layered contaminated soil is derived, which can be further used to describe the average degree of diffusion. The reliability and accuracy of the developed solution is verified by comparing with the existing analytical solution and numerical simulation results. Selected numerical examples are further presented to analyze the influence of the imperfect diffusion boundaries on the spatial/time distribution of contaminant concentration, average degree of diffusion and mass flux. The results show that greater imperfect diffusion capacity coefficients lead to lower contaminant concentration distribution within the entire depth range and higher average degree of diffusion for a given time. Particularly, it only takes 23.32 years to complete the entire diffusion process when imperfect diffusion capacity coefficients are infinite. The developed solution can provide useful guidance for engineering practice.
\end{abstract}

Keywords: Analytical solution, Average degree of diffusion, Concentration, Double-layered contaminated soil, Imperfect diffusion boundaries.

\section{Introduction}

As an economical and effective treatment method, contaminant repository is mainly applied into the disposal of industrial pollution, landfills and tailings, and the stock of various these pollutants is over $2.82 \times 10^{10}$ ton per year [1]. The engineering problem of the repository as a source of contaminant located below the water source or above the aquifer is one of the most important challenges. Natural weak diffusive clay layer or geosynthetic layer with slight diffusive capabilities is commonly used to cover underwater contaminant sources [2], such as the stratified contaminated soil layer containing a large amount of high-concentration and highly corrosive leachate. It should be pointed out that under the coupling effect of multiple fields, the contaminant source of the repository is likely to diffuse to the adjacent soil and water with great difficulty to repair again, which seriously threatens the environmental health [3-5]. In the above cases, molecular diffusion induced by concentration differences is the key factor for the contaminant diffusion to the adjacent soil or cover layer because of its low hydraulic conductivity of the clay (e.g., $<10^{-7} \mathrm{~cm} / \mathrm{s}$ ), low leachate head (e.g., $<0.3 \mathrm{~m}$ ), and small Peclet number [6-9]. Besides, diffusive transport problem comprehensively exists in the engineering materials, such as compacted clay liner [10, 11], underwater cap [12], geosynthetic clay lining [13], and composite liners $[14,15]$. In order to prevent and slow down the damage of contaminant on the surrounding environment, it is necessary to study the related problems [16-18] caused by the diffusion of contaminants.

Although the diffusion problem in engineering practice can be analyzed by the numerical simulation schemes [19, 20], analytical methods can provide a deep understanding for the essence of the related problem. In addition, the derived analytical solutions are

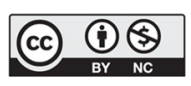

This is an Open Access article distributed under the terms of the Creative Commons Attribution Non-Commercial License (http://creativecommons.org/licenses/by-nc/3.0/) which permits unrestricted non-commercial use, distribution, and reproduction in any medium, provided the original work is properly cited.

Copyright (c) 2022 Korean Society of Environmental Engineers
Received January 28, 2021 Accepted June 02, 2021

${ }^{\dagger}$ Corresponding author

E-mail: zhangzhiqing2000@163.com; wangzsd@zjut.edu.cn

Tel: +86-13858110682 Fax: +86-57163743301

ORCID: 0000-0002-9331-4302(Zhang), 0000-0003-4065-5960(Wang) 
simple, economical and effective, and they can also be used to validate the complex numerical simulation schemes in which it is difficult to accurately determine the valid parameters [21-23]. For instance, for the preliminary design of a landfill site, most of the valid parameters are difficult to obtain directly, but the analytical solutions can lead to a smooth design [24-27]. Over the past many years, various analytical methods are proposed to solve the contaminant diffusion problem of different types of porous media by supposing different diffusion boundaries and initial conditions. For instance, Foose et al. [14] introduced the Laplace transform to derive the analytical solution of the contaminant diffusion in a double-layered medium considering the semi-infinite boundary, and further used the finite difference method to study the one-dimensional diffusion of the double-layered medium under the assumption of zero-concentration boundary. Shackelford et al. [28] adopted the methodology of transforming the analytical solution of small strain one-dimensional consolidation of saturated compressible porous medium into the solution of one-dimensional liquid-phase diffusion through saturated porous medium under time-dependent boundary and complex initial conditions. Chen et al. [29], Xie et al. [30] proposed the analytical solutions for the one-dimensional diffusion of contaminant in soil considering the boundary of finite thickness and the stratification of soil media. $\mathrm{Li}$ et al. [31] established the solute convection-diffusion equation in a double-layered finite depth porous medium, and developed the analytical solutions corresponding to different boundary conditions. It can be observed from the above studies that the description of the boundary is assumed to be an ideal and perfect one.

However, the diffusion from contaminated soil to the adjacent materials are really not the ideal diffusion due to the great difference in the diffusive behaviors between different materials. It is also reported that the damage on the barrier layer and technical defects during construction and operation are inevitable, which increases the corresponding average diffusion capability [32, 33]. Therefore, in order to better portray this kind of complicated problem, the interface between the contaminated soil layers and the adjacent layers could be modeled as the imperfect diffusion boundaries in theory, which motivates the present study.

In this paper, we propose a novel one-dimensional model for the diffusion of contaminants from the double-layered contaminated soil layers to the adjacent capping and bottom natural soil layers with imperfect diffusion boundaries. It is noted that the newly developed imperfect diffusion boundary is used to portrait the imperfect diffusion behavior from the contaminated soil to the adjacent layers. The diffusion equation of contaminant is split into two separate ordinary differential equations using separation of variables method, and the corresponding general solutions are obtained. After substituting the initial conditions, continuity conditions and imperfect boundary conditions of the layered system into the general solutions, and using the orthogonality of trigonometric functions, the analytical solution for the diffusion of contaminant is derived. The developed solutions are verified by comparing with the existing analytical solution and numerical results by the COMSOL Multiphysics software. The influence of the imperfect diffusion boundaries on the spatial/time distribution of contaminant concentration, average degree of diffusion and mass flux are also analyzed in details. The analytical solutions proposed in this paper can be served as the benchmarks for the complicated and powerful numerical solutions and provide useful guidelines for engineering practice.

\section{Solution of the Contaminant Transport Problem}

\subsection{Basic Assumptions and Mathematical Model}

The following assumptions are adopted when establishing a one-dimensional contaminant diffusion model from a double-layered contaminated soil to the capping and bottom natural soil layers:

Hydraulic conduction, degradation, convection-dispersion, adsorption retardation, and other transport mode on the concentration of contaminants is not considered as the way of contaminant transport, while the main transport mode of contaminants in the soil is molecular diffusion.

The top and bottom boundaries of the contaminated soils are modelled as imperfect diffusion boundaries. The outlet boundaries of the capping layer and bottom natural soil layer are assumed to be zero concentration, i.e., full diffusion boundary.

Each contaminated soil layer is homogeneous, isotropic and saturated soil, and the corresponding physical properties (e.g., porosity, effective diffusion coefficient, etc.) are assumed to be constants.

The diffusion of contaminants in the contaminated soils is only along the depth direction (i.e., one-dimensional diffusion), and follows Fick's second law.

The concentration of contaminant and flux are continuous at the interface between two contaminated soil layers.

The contaminants diffusion from the contaminated soils to the top capping layer and the bottom natural soil layer with imperfect diffusion boundaries is illustrated in Fig. 1. A double-layered contaminated soils is over the bottom natural soil layer, and the top capping layer is covered on it to prevent contaminant entering into the water. The thickness of the top cap layer, contaminated soil layers 1 and 2, and the bottom healthy soil layer are denoted by $L_{1}, h_{1}, h_{2}$ and $L_{2}$, respectively. The origin of the coordinate is at the top surface of contaminated soil layer 1 with the positive $z$ direction being downwards. The interfaces between the contaminated soil layers and the adjacent layers are modelled as the imperfect boundaries, i.e., the concentration at the boundaries between the contaminated soil layers and the adjacent layers is continuous, but the diffusion rate is discontinuous. With this kind of imperfect boundaries, not only can various soil layers with different diffusion capabilities be handled, but also can be reduced to the classical engineering problems. For instance, when the bottom boundary has zero diffusivity and the top diffusivity is weak, it can be transformed into a boundary condition commonly used in landfill storage problems. When the top and bottom of the contaminated layers are full diffusion boundary, it can be transformed into the boundary condition used for the problem of the contaminated soil diffusing to the adjacent water source. 


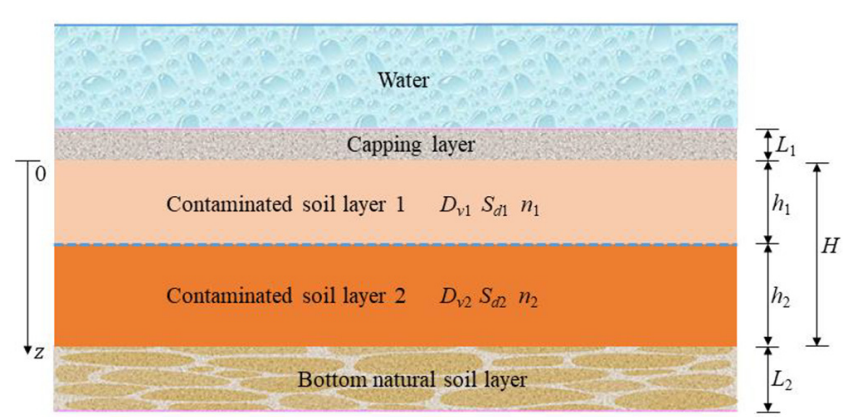

Fig. 1. Diagrammatic sketch for one-dimensional diffusion model of double-layered contaminated soil with imperfect boundaries.

The governing equation for the contaminant diffusion from the double-layered contaminated soil to the adjacent layer can be written as

$$
\frac{\partial C_{i}(z, t)}{\partial t}=D_{v i} \frac{\partial^{2} C_{i}(z, t)}{\partial z^{2}}
$$

where $C_{i}(z, t)$ and $D_{v i}$ are the concentration and effective diffusion coefficient of contaminant in the $i$ th $(i=1,2)$ contaminated soil layer, respectively; For the ith layer, the effective diffusion coefficient $D_{v i}$ can be further defined as the product of the apparent tortuosity factor $\tau_{a i}$ and the aqueous diffusion coefficient for the solutes $D_{0 i}$ i.e., $D_{v i}=D_{0 i} \tau_{a i}[31]$; $z$ is the spatial coordinate variable along the depth direction, and $t$ is the time variable.

The initial concentration of the contaminant in the contaminated soil layers can be expressed as

$$
C_{1}(z, t=0)=C_{2}(z, t=0)=C_{0}
$$

where $C_{0}$ is the initial concentration of contaminant in contaminated soil layers 1 and 2 when $t=0$.

Given that the contaminant concentration and flux are continuous at $z=h_{1}$, hence the following continuity conditions hold

$$
\begin{gathered}
C_{1}\left(h_{1}, t\right)=C_{2}\left(h_{1}, t\right) \\
n_{1} D_{v 1} \frac{\partial C_{1}\left(h_{1}, t\right)}{\partial z}=n_{2} D_{v 2} \frac{\partial C_{2}\left(h_{1}, t\right)}{\partial z}
\end{gathered}
$$

where $n_{1}$ and $n_{2}$ are the porosities of the contaminated soil layers 1 and 2 , respectively.

The concentration at the boundary between the contaminated soil layers and the adjacent layers is continuous, but the diffusion rate is discontinuous. So the imperfect diffusion boundary conditions at the top surface of contaminated soil layer 1 (i.e., $z=$ 0 ) and the bottom surface of the contaminated soil layer 2 (i.e., $z=H$ ) can be written as

$$
\begin{gathered}
\frac{\partial C_{1}(z=0, t)}{\partial z}=\frac{S_{d 1}}{H} C_{1}(z=0, t) \\
\frac{\partial C_{2}(z=H, t)}{\partial z}=-\frac{S_{d 2}}{H} C_{2}(z=H, t)
\end{gathered}
$$

in which

$$
S_{d 1}=\frac{D_{1} H}{D_{v 1} L_{1}} ; S_{d 2}=\frac{D_{2} H}{D_{v 2} L_{2}}
$$

where $D_{1}\left(L_{1}\right)$ and $D_{2}\left(L_{2}\right)$ are, respectively, the effective diffusion coefficients (thickness) of the top capping layer and the bottom natural soil layer; $S_{d 1}$ and $S_{d 2}$ are the imperfect diffusion capacity coefficients representing the ratio of the diffusion capacity of the imperfect diffusion layers (i.e., the top capping layer and the bottom natural soil layer).

It is noted that Eq. (5) and Eq. (6) describes the continuity condition of the top and bottom boundaries of the contaminated soil layer, and the boundary concentration is controlled by the imperfect diffusion capacity coefficients $S_{d i}(i=1,2)$. When the capping layer has perfect protective function (ideal condition), it can be considered that it has no ability to diffuse, i.e, $S_{d 1}=0$. However, when the protective function of capping layer is relatively weak, it has certain ability to diffuse, i.e., $S_{d 1}>0$. The above description also applies to the bottom natural soil layer.

\subsection{General Solution}

\subsubsection{Concentration in contaminated soil}

According to the separation of variables method, the following single-variable function $C_{i}(z, t)$ is introduced

$$
C_{i}(z, t)=\phi_{i}(z) \theta_{i}(t), \quad i=1,2
$$

Substituting Eq. (8) into Eq. (1) and dividing both sides of the equations by $\phi_{i}(z) \theta_{i}(t)$ at the same time results in

$$
\frac{1}{D_{v i} \theta_{i}(t)} \frac{\mathrm{d} \theta_{i}(t)}{\mathrm{d} t}=\frac{1}{\phi(z)} \frac{\mathrm{d}^{2} \phi_{i}(z)}{\mathrm{d} z^{2}}, \quad i=1,2
$$

Introducing the constant $\lambda$, Eq. (9) can be split into two separate ordinary differential equations as

$$
\begin{gathered}
\frac{1}{D_{v i} \theta_{i}(t)} \frac{\mathrm{d} \theta_{i}(t)}{\mathrm{d} t}=-\lambda \\
\frac{1}{\phi_{i}(z)} \frac{\mathrm{d}^{2} \phi_{i}(z)}{\mathrm{d} z^{2}}=-\lambda
\end{gathered}
$$

The solutions of Eq. (10) and Eq. (11) can be expressed as

$$
\begin{gathered}
\theta_{i}(t)=A_{i} \mathrm{e}^{-\lambda D_{v i} t} \\
\phi_{i}(z)=B_{i} \cos (\sqrt{\lambda} z)+F_{i} \sin (\sqrt{\lambda} z) \\
\mathrm{d} \phi_{i}(z) / \mathrm{d} z=-B_{i} \sqrt{\lambda} \sin (\sqrt{\lambda} z)+F_{i} \sqrt{\lambda} \cos (\sqrt{\lambda} z)
\end{gathered}
$$

Substituting the initial conditions, continuity conditions and imperfect diffusion boundary conditions given in Eqs. (2)-(6) into 
Eqs. (12)-(14), and utilizing the orthogonality of trigonometric functions, we have

$$
\begin{gathered}
C_{1}=C_{0} \sum_{m=1}^{\infty} f_{m}\left[\sin \left(\frac{\lambda_{m}}{H} z\right)+\frac{\lambda_{m}}{S_{d 1}} \cos \left(\frac{\lambda_{m}}{H} z\right)\right] \mathrm{e}^{-\lambda_{m}^{2} T_{v}}, 0 \leq z \leq h_{1} \\
C_{2}=C_{0} \sum_{m=1}^{\infty} f_{m} Q_{m}\left[j \lambda_{m} \cos \left(j \lambda_{m} \frac{H-z}{H}\right)+S_{d 2} \sin \left(j \lambda_{m} \frac{H-z}{H}\right) \mathrm{e}^{-\lambda_{m}^{2} T_{v}},\right. \\
h_{1} \leq z \leq H
\end{gathered}
$$

in which

$$
\begin{gathered}
f_{m}=\frac{2 S_{d 1}^{2}\left(1+\sqrt{a b} S_{d 2} Q_{m}\right)}{\lambda_{m}\left\{k\left(\lambda_{m}^{2}+S_{d 1}^{2}\right)+S_{d 1}+b S_{d 1}^{2} Q_{m}^{2}\left[(1-k)\left(j^{2} \lambda_{m}^{2}+S_{d 2}^{2}\right)+S_{d 2}\right]\right\}} \\
Q_{m}=\frac{S_{d 1} \cos \left(k \lambda_{m}\right)-\lambda_{m} \sin \left(k \lambda_{m}\right)}{\sqrt{a b} S_{d 1}\left\{j \lambda_{m} \sin \left[(1-k) j \lambda_{m}\right]-S_{d 2} \cos \left[(1-k) j \lambda_{m}\right]\right\}} \\
a=\frac{n_{2} D_{v 2}}{n_{1} D_{v 1}} ; b=\frac{n_{2}}{n_{1}} ; k=\frac{h_{1}}{H} ; j=\sqrt{D_{v 1} / D_{v 2}}=\sqrt{b / a} ; T_{v}=\frac{D_{v 1} t}{H^{2}}
\end{gathered}
$$

where $\lambda_{m}(m=1,2,3, \ldots)$ is the positive root of the following characteristic equation

$$
\begin{gathered}
\sqrt{a b}\left(\lambda_{m}+S_{d 1} \tan \left(k \lambda_{m}\right)\left\{j \lambda_{m} \tan \left[j(1-k) \lambda_{m}\right]-S_{d 2}\right\}\right. \\
-\left[S_{d 1}-\lambda_{m} \tan \left(k \lambda_{m}\right)\right]\left\{j \lambda_{m}+S_{d 2} \tan \left[j(1-k) \lambda_{m}\right]\right\}=0
\end{gathered}
$$

It is noted that $\lambda_{m}$ in Eq. (19) can be solved using the dichotomization method. Besides, the series in Eq. (15) converge quickly, and the accuracy of the series can be ensured when setting $m$ $=100$.

The mass flux in the $i$ th $(i=1,2)$ layer caused by diffusion in contaminated soil can be expressed in terms of the first Fick's law as

$$
J_{i}(z, t)=-n_{i} D_{v i} \frac{\partial C_{i}(z, t)}{\partial z}
$$

where $J_{i}(z, t)$ is the mass flux in the $i$ th contaminated soil layer.

Substituting Eq. (20) into Eq. (15) results in

$$
\begin{gathered}
J_{1}(z, t)=-n_{1} D_{v 1} C_{0} \sum_{m=1}^{\infty} f_{m}\left[\frac{\lambda_{m}}{H} \cos \left(\frac{\lambda_{m}}{H} z\right)-\frac{\lambda_{m}^{2}}{S_{d 1} H} \sin \left(\frac{\lambda_{m}}{H} z\right)\right] \mathrm{e}^{-\lambda_{m}^{2} T_{v}}, \\
0 \leq z \leq h_{1} \\
J_{2}(z, t)=-n_{2} D_{v 2} C_{0} \sum_{m=1}^{\infty} f_{m} Q_{m}\left[\frac{j^{2} \lambda_{m}^{2}}{H} \sin \left(j \lambda_{m} \frac{H-z}{H}\right)-\frac{S_{d 2} j \lambda_{m}}{H} \cos \left(j \lambda_{m} \frac{H-z}{H}\right)\right] \mathrm{e}^{-\lambda_{m}^{2} T_{v}}, \\
h_{1} \leq z \leq H
\end{gathered}
$$

\subsubsection{Average degree of diffusion}

Since the contaminant concentrations in the two contaminated soil layers are derived in the above section, the degree of diffusion of contaminants can be further determined. For the convenience of the following analysis, the average degree of diffusion $D_{a}$ is defined as the ratio of the average concentration diffused to the adjacent layer to its initial concentration as [7].

$$
\begin{aligned}
D_{a} & =\frac{n_{1} \int_{0}^{h_{1}}\left(C_{0}-C_{1}\right) \mathrm{d} z+n_{2} \int_{h_{1}}^{H}\left(C_{0}-C_{2}\right) \mathrm{d} z}{C_{0}\left(n_{1} h_{1}+n_{2} h_{2}\right)} \\
& =\frac{k D_{d 1}+b(1-k) D_{d 2}}{k+b(1-k)} \\
& =1-\sum_{m=1}^{\infty} f_{m} \frac{1+\sqrt{a b} S_{d 2} Q_{m}}{\lambda_{m}[k+b(1-k)]} \mathrm{e}^{-\lambda_{m}^{2} T_{v}}
\end{aligned}
$$

in which

$$
\begin{aligned}
& D_{d 1}=1-\frac{\frac{1}{h_{1}} \int_{0}^{h_{1}} C_{1} \mathrm{~d} z}{C_{0}}=1-\sum_{m=1}^{\infty} f_{m} \frac{S_{d 1}\left[1-\cos \left(k \lambda_{m}\right)\right]+\lambda_{m} \sin \left(k \lambda_{m}\right)}{S_{d 1} k \lambda_{m}-\lambda_{m}^{2} T_{v}} \\
& D_{d 2}=1-\frac{\frac{1}{h_{2}} \int_{h_{1}}^{H} C_{2} \mathrm{~d} z}{C_{0}}=1-\sum_{m=1}^{\infty} f_{m} \frac{S_{d 2}\left\{1-\cos \left[j(1-k) \lambda_{m}\right]\right\}+\lambda_{m} \sin \left[j(1-k) \lambda_{m}\right]}{(1-k) j \lambda_{m}} Q_{m} \mathrm{e}^{-\lambda_{m}^{2} T_{m}}
\end{aligned}
$$

where $D_{d i}(i=1,2)$ is the average degree of diffusion of contaminant in the ith contaminated soil layer; $D_{a}$ is the average degree of diffusion, which describes the overall degree of diffusion of contaminants in the contaminated soil layers.

It is noted that $S_{d i}=0$ and $\infty(i=1,2)$ are the ideal boundaries, which correspond to the full diffusion and non-diffusion boundaries, respectively. When $S_{d i}$ are equal to the finite value, the boundaries are really the imperfect boundaries. When $S_{d 1}$ is equal to 0 and the value of $S_{d 2}$ represents the diffusion capacity of the boundary between the liner and the contaminated soil layer, the calculation model can be transformed into the corresponding engineering problem in the landfill $[11,13]$. When $S_{d 2}$ is equal to 0 and the value of $S_{d 1}$ represents the diffusion capacity of the boundary between the capping layer and the contaminated soil layer, the calculation model can be transformed into the engineering problem corresponding to the underwater capping layer [12]. In addition, the diffusion capacity of the boundary layer increases with the increase of $S_{d i}$.

\section{Results and Discussion}

\subsection{Verification of the Present Solution}

In the past study, Li and Cleall [31] developed general analytical solutions for conservative solute diffusion in one-dimensional double-layered porous media, and analyzed the contaminant diffusion in a reduced single-layer uncapped contaminated sediment with bottom zero-flux boundary. In order to verify the reliability and accuracy of the analytical solution proposed in the present paper, the reduced present solution is compared with this existing solution first. The original parameters used in $\mathrm{Li}$ and Cleall [31] are: $H$ $=1.5 \mathrm{~m}, D_{V}=9.4 \times 10^{-10} \mathrm{~m}^{2} / \mathrm{s}, n=0.45$ and retardation factor 


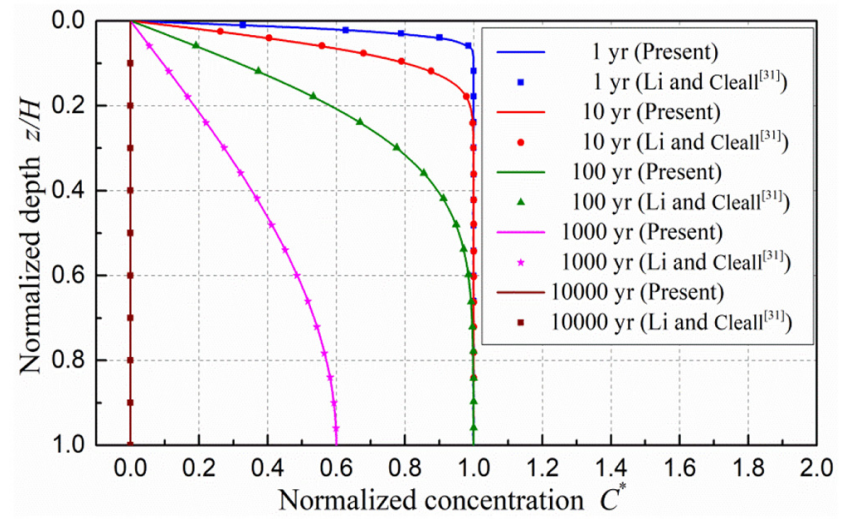

Fig. 2. Variation of normalized contaminant concentration $C^{*}$ with normalized depth $z / H$. Present solution vs. Li and Cleall's solution.

$R_{d}=43.3$. It is noted that $\mathrm{Li}$ and Cleall [31] considered the effect of retardation factor, hence we need to modify $D_{v}$ as $D_{v} / R_{d}$ to keep the same condition. Hence, the input parameters in the present calculation are: $h_{1}=h_{2}=0.75 \mathrm{~m}, D_{v 1}=D_{v 2}=2.1709 \times 10^{-11}$ $\mathrm{m}^{2} / \mathrm{s}, n_{1}=n_{2}=0.45$. For general applications, the normalized contaminant concentration is defined as $C^{*}=C / C_{0}$, in which $C$ denotes the concentration of contaminant in contaminated layers 1 and 2. Fig. 2 shows the comparison of contaminant concentration vs. normalized depth between the reduced present solution and $\mathrm{Li}$ and Cleall's solution. It can be seen from Fig. 2 that the results from the present solution are very close to those from Li and Cleall [31] for different times.

To further verify the reliability of the present solution, we compare the present analytical solution with two numerical examples calculated by COMSOL Multiphysics software. The contaminated soil layer and the imperfect diffusion layer system are assumed to be layered porous media with different effective diffusion coefficients in the following calculation. The contaminated soil layers 1 and 2 contain, respectively, $\mathrm{ZnSO}_{4}$ and $\mathrm{NaCl}$ contaminant sources with the same initial contaminant concentration. The effective diffusion coefficients of $\mathrm{Zn}^{2+}$ (i.e., $D_{V 1}$ ) and $\mathrm{Cl}^{-}$(i.e., $D_{V 2}$ ) are employed in the following calculation [35]. Four cases with different combination of $S_{d 1}$ and $S_{d 2}$ are: Case 1 with $S_{d 1}=\infty, S_{d 2}=\infty$; Case 2 with $S_{d 1}=\infty, S_{d 2}=1$; Case 3 with $S_{d 1}=15, S_{d 2}=0$; Case 4 with $S_{d 1}=5, S_{d 2}=0$. Unless otherwise specified, the basic parameters used in the following calculations (i.e., Fig. 3 to Fig. 8) are: $H=1 \mathrm{~m}, h_{1}=0.5 \mathrm{~m}, T_{V}=0.129$ or $0.279, C_{0}=0.1 \mathrm{~mol} / \mathrm{L}, D_{V 1}$ $=3.3 \times 10^{-10} \mathrm{~m}^{2} / \mathrm{s}, D_{v 2}=6.8 \times 10^{-10} \mathrm{~m}^{2} / \mathrm{s}, n_{1}=0.25, n_{2}=0.5$. It should be pointed out again that $S_{d i}=0, \infty$ and finite values $(i=1,2)$ represent the non-diffusion, full diffusion and imperfect diffusion boundaries.

As for the following numerical simulation, the first numerical example (i.e., numerical example A) corresponds to the same assumption condition with the present analytical solution, and the second numerical example (i.e., numerical example B) further considers the maximum contribution of advection and mechanical dispersion to diffusion-controlled transport. It is noted again that under the condition of low head, permeability coefficient and Peclet number, the transport of contaminant is diffusion-controlled [6-9]. When considering the role of advection and mechan- ical dispersion in the process of diffusion-controlled transport, the hydrodynamic dispersion coefficient $D_{r i}$ in the ith layer can be defined as [36].

$$
D_{r i}=D_{v i}+D_{m i}
$$

where $D_{m i}$ is the mechanical dispersion coefficient in the $i$ th layer ( $i=1,2)$, and it can be defined as $D_{m i}=h_{i} V_{i} / 10$ with $h_{i}$ and $v_{i}$ being the thickness and seepage velocity of the ith layer, respectively.

Darcy flow rate of a double-layered system can be defined as

$$
v_{a}=\frac{K_{a}\left(H_{w}+H\right)}{H}=n_{i} v_{i}
$$

where $K_{a}$ is the harmonic average of the permeability coefficient in each layer [36]; $H_{W}$ and $n_{i}$ are, respectively, the water head height and porosity of the $i$ th layer $(i=1,2)$.

To reflect the maximum advection and mechanical dispersion contribution to the diffusion-controlled transport, the critical values of permeability coefficient and water head of each layer are selected with satisfying $v_{i} h_{i} / D_{v i} \leq 1$ [37]. The additional selected parameters used in numerical example B are: $H_{w}=0.3 \mathrm{~m}, K_{a}=1.27 \times 10^{-10}$ $\mathrm{m} / \mathrm{s}, v_{1}=6.6 \times 10^{-10} \mathrm{~m} / \mathrm{s}, v_{2}=3.3 \times 10^{-10} \mathrm{~m} / \mathrm{s}$.

Fig. 3 shows the comparison of the normalized contaminant concentration distribution along depth direction between the present solution and numerical solution. It can be seen from Fig. 3 that under different working conditions, the results from the present solution generally agree well with those in numerical example A, which further validates the reliability and accuracy of the present solution. It can be also observed from Fig. 3 that molecular diffusion still controls the transport of contaminant in numerical example B. When considering the maximum contribution of advection and mechanical dispersion, the normalized concentration $C^{*}$ in numerical example B is smaller than that in numerical example A.

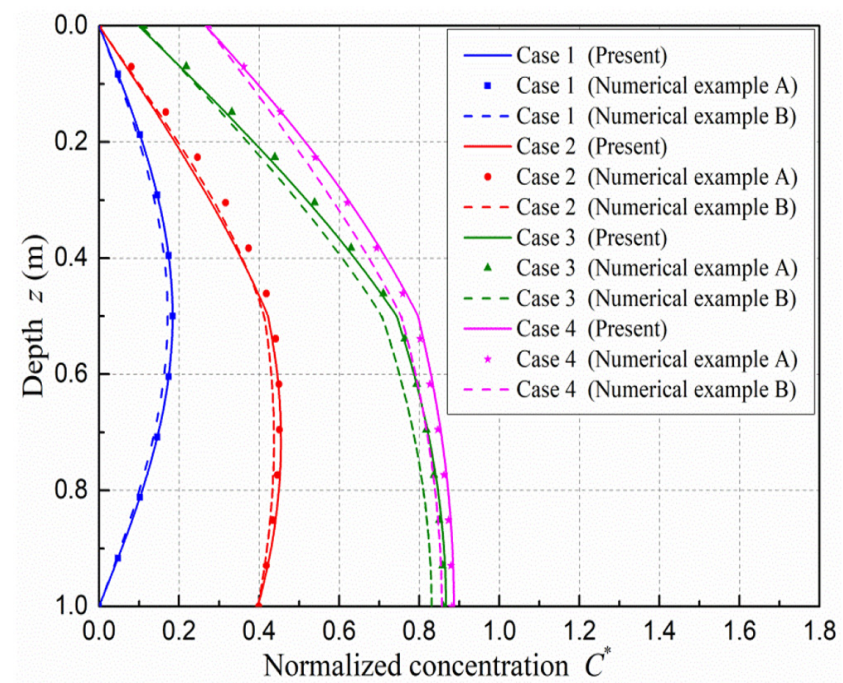

Fig. 3. Variation of normalized contaminant concentration $C^{*}$ with depth z. Present solution vs. numerical solution $\left(T_{v}=0.279\right.$ (i.e., $t$ $=13.0103$ years)). 
Moreover, as the imperfect diffusion capacity coefficients increases, the convection and mechanical dispersion contributes less to transport of contaminant.

\subsection{Influence of Imperfect Diffusion Boundaries}

In this section, we mainly analyze the influence of imperfect diffusion boundaries on the contaminant concentration, the average degree of diffusion and mass flux. The influence of the imperfect diffusion boundaries on the distribution of the normalized contaminant concentration distribution along the depth direction is shown in Fig. 4. The behavior of the imperfect diffusion boundaries is presented by the imperfect diffusion capacity coefficients (i.e., $S_{d 1}$ and $\left.S_{d 2}\right)$. It can be observed from Fig. 4 that $S_{d i}(i=1,2)$ have great influence on the concentration distribution along the depth direction. When $S_{d i}=0$ (i.e., ideal non-diffusion boundary), the normalized contaminant concentration $C^{*}$ is equal to 1 , which means that no contaminant diffuses to the adjacent layer. However, this is very difficult to realize in the engineering practice. When $S_{d i}=\infty$ (i.e., ideal full diffusion boundary), the concentrations at $z=0$ and $H$ are equal to zero, indicating that the contaminants at the top surface of the contaminated soil layer 1 and at the bottom surface of the contaminated soil layer 2 can freely transport to the top capping and bottom natural soil layers. Hence, in this case (i.e., $S_{d i}=\infty$ ), $C^{*}$ is the smallest when $t=6.0155$ years. As $S_{d 1}$ or $S_{d 2}$ decreases, $C^{*}$ shows marked increase within the entire depth range. This indicates that the diffusion capacity of the capping and bottom natural soil layers decreases markedly with decreasing $S_{d 1}$ or $S_{d 2}$, and more contaminants are kept in the contaminated soil layers. It can be also seen from Fig. 4 that there exists obvious change of curve slope at the interface of two contaminated soil layers. The greater the difference between the values of $S_{d 1}$ and $S_{d 2}$, the more obvious the curve slope changes at the interface.

Fig. 5 describes the influence of imperfect diffusion boundaries on the time history of normalized contaminant concentration at a fixed depth. It can be seen from Fig. 5 that when time factor $T_{V}$ is relatively small, the contaminant concentration at fixed level

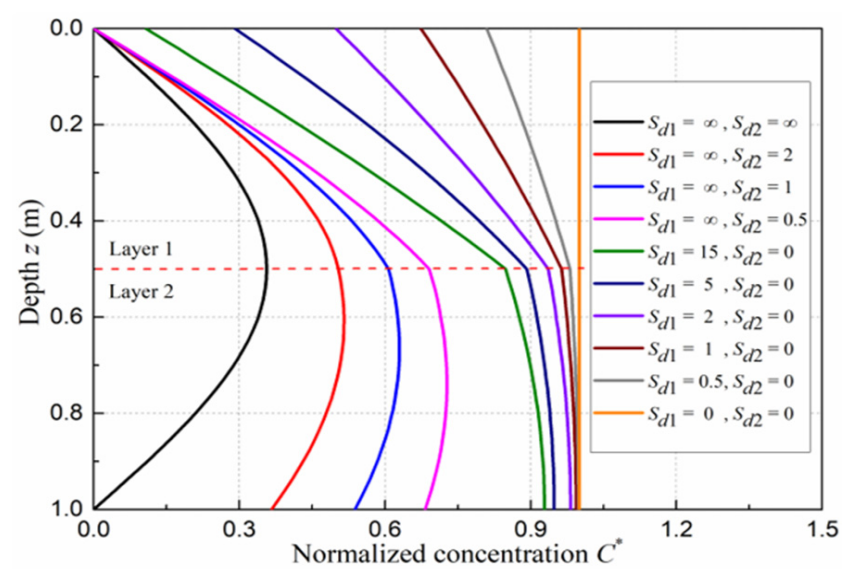

Fig. 4. Effect of imperfect diffusion boundaries on the distribution of normalized concentration along depth direction $\left(T_{v}=0.129\right.$ (i.e., $t=6.0155$ years)). (i.e., $z=0.5 \mathrm{~m}$ ) decreases greatly with increasing $S_{d i}$, especially when $S_{d i}=\infty$. It can be also observed from Fig. 5 that the decreasing ratio of the contaminant concentration in the contaminated soil decreases with increasing time factor. This phenomenon is in accordance with the basic law, i.e., the diffusion velocity of contaminant is affected by the concentration gradient.

Fig. 6 depicts the influence of imperfect diffusion boundaries on the variation of time history of the average degree of diffusion. The $x$-axis is in a base-10 logarithmic scale. It can be observed from Fig. 6 that the average degree of diffusion $D_{a}$ shows marked increase as time increases. As the time further increases, the changing rate of the average degree of diffusion decreases due to the decrease of the concentration gradient (see the upper four curves in Fig. 6). It can be also seen from Fig. 6 that for a given time, the larger the value of the combination of $S_{d i}$, the greater the value of the average degree of diffusion $D_{a}$. When $S_{d 1}=S_{d 2}=\infty$, the top and bottom surfaces of the contaminated soil layers are ideally full diffusion boundaries, and completing the diffusion process

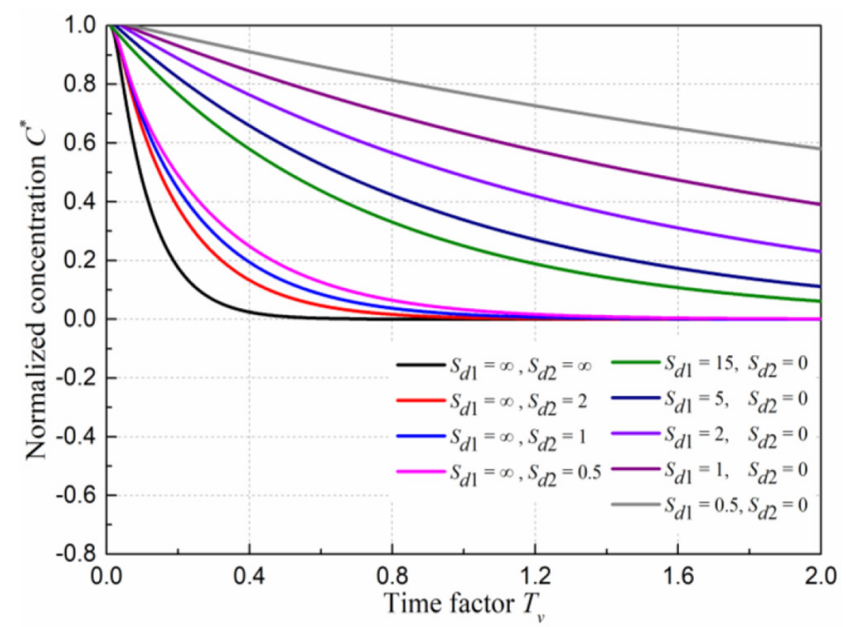

Fig. 5. Effect of imperfect diffusion boundaries on the time history of normalized concentration $(z=0.5 \mathrm{~m})$.

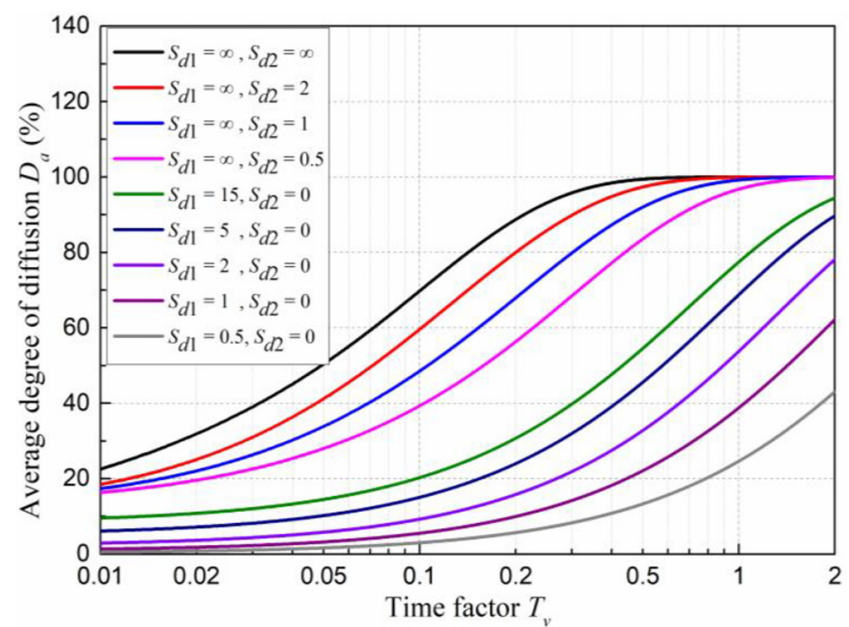

Fig. 6. Effect of imperfect diffusion boundaries on the time history of average degree of diffusion. 


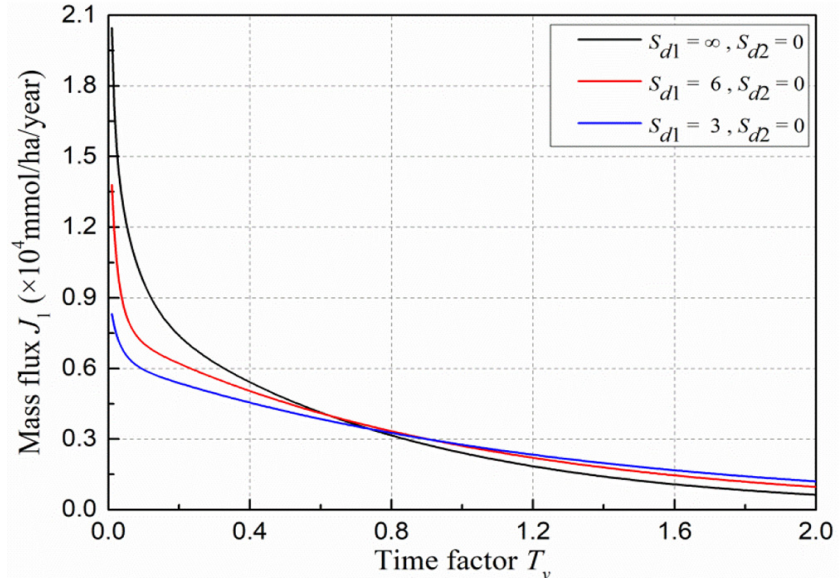

Fig. 7. Effect of imperfect diffusion boundaries on the time history of mass flux at $z=0$ level.

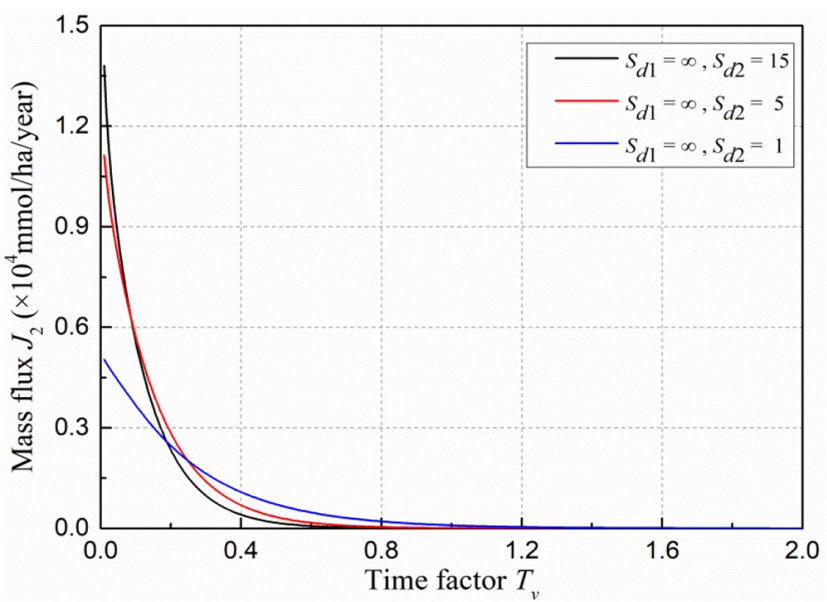

Fig. 8. Effect of imperfect diffusion boundaries on the time history of mass flux at $z=H$ level.

needs only 23.32 years. However, when $S_{d 2}=0$, due to the influence of the ideally non-diffusion boundary of contaminated soil layer 2 , the contaminant can only diffuse through the top capping layer. Accordingly, it needs at least 69.95 years to complete the entire diffusion process. It can be concluded from Fig. 4 Fig. 6 that the imperfect diffusion capacity coefficients have great effect on the diffusion of the contaminant. Therefore, in engineering practice, the intrinsic diffusion capacity of the capping layer should be carefully designed for the better environmental conservation.

Fig. 7 and Fig. 8 depict, respectively, the influence of the imperfect diffusion boundaries on the time history of mass flux at $z=0$ and $z=H$ level. It can be observed from Fig. 7 and Fig. 8 that the imperfect diffusion boundaries have great influence on the variation of the mass flux with increasing time. In the initial stage of the diffusion (i.e., $T_{V}$ is relatively small), the mass flux at the top and bottom level of the contaminated soil layers increases with the increase of $S_{d 1}$ or $S_{d 2}$. However, when the time factor $T_{V}$ further increases, the corresponding mass flux decreases with the increase of $S_{d 1}$ or $S_{d 2}$. The main reason for this phenomenon is that in the initial stage, the contaminant concentration in the soil decreases quickly for a larger $S_{d 1}$ or $S_{d 2}$ value, which induces the decreasing concentration difference in contaminated soil. As a result, when the time is relatively big, the mass flux corresponding to larger $S_{d 1}$ or $S_{d 2}$ value is smaller than that corresponding to smaller $S_{d 1}$ or $S_{d 2}$ value.

\section{Conclusions}

In this paper, we propose an analytical method to solve the one-dimensional diffusion of contaminants from the double-layered contaminated soil layers to the adjacent capping and bottom natural soil layers with imperfect diffusion boundaries. At first, the governing equation of contaminant diffusion is split into two ordinary differential equations via the separation of variables method. Then the general solutions for the decoupled ordinary differential equations are obtained. By utilizing the initial conditions, continuity conditions, imperfect boundary conditions of the layered system, and the orthogonality of trigonometric functions, the analytical solution for the contaminant diffusion is finally derived through some algebraic operations. Through the comparison with the existing analytical solution and the numerical solution, the reliability and accuracy of the present solution is validated, and the influence of advection and mechanical dispersion is analyzed. Finally, the influence of the imperfect boundaries (represented by the imperfect diffusion capacity coefficients $S_{d 1}$ and $S_{d 2}$ ) on the spatial/time distribution of contaminant concentration, average degree of diffusion and the mass flux is analyzed. The theory developed in this paper reveals the diffusion mechanism of the contaminant from the double-layered contaminated soil layers to the adjacent layers considering the influence of the imperfect diffusion boundaries, which can provide useful guidance for the engineering practice and could be further served as benchmarks for future numerical solutions. The main conclusions are as follows:

For the diffusion-controlled transport of contaminant, the contribution of advection and mechanical dispersion to the transport process is relatively small. The influence of advection and mechanical dispersion decreases with the increase of imperfect diffusion capacity coefficients $S_{d 1}$ and $S_{d 2}$.

Imperfect diffusion capacity coefficients have great influence on the spatial/time distribution of contaminant concentration. As $S_{d 1}$ or $S_{d 2}$ decreases, contaminant concentration shows marked increase within the entire depth range for a given time. There also exists obvious change of curve slope at the interface of two contaminated soil layers due to the layering of the soil. When time factor $T_{V}$ is relatively small, the contaminant concentration at a fixed depth of the contaminated soil layer decreases greatly with increasing $S_{d 1}$ or $S_{d 2}$ for a given time.

Imperfect diffusion capacity coefficients $S_{d 1}$ and $S_{d 2}$ have great influence on the average degree of diffusion and mass flux. The average degree of diffusion increases with increasing $S_{d 1}$ or $S_{d 2}$ for a given time. The mass flux decreases greatly with increasing $S_{d 1}$ or $S_{d 2}$ in the initial stage, while it increases with increasing $S_{d 1}$ or $S_{d 2}$ when time factor $T_{V}$ is relatively large. 


\section{Nomenclature}

$C_{0} \quad$ Initial concentration of contaminant in contaminated soil layers 1 and 2

$C_{i} \quad$ Concentration of contaminant in the ith contaminated soil layer

$C^{\star} \quad$ Normalized concentration of contaminant

$D_{1} \quad$ Effective diffusion coefficient in capping layer

$D_{2} \quad$ Effective diffusion coefficient in bottom natural soil layer

$D_{a} \quad$ Average degree of diffusion

$D_{d i} \quad$ Average degree of diffusion of contaminant in the ith contaminated soil layer

$D_{v i} \quad$ Effective diffusion coefficient in the $i$ th contaminated soil layer

$J_{i} \quad$ Mass flux in the $i$ th contaminated soil layer

$n_{i} \quad$ Porosities of the ith contaminated soil layer

$S_{d i} \quad$ Imperfect diffusion capacity coefficients in ith contaminated soil layer

$t \quad$ Time variable

$T_{V} \quad$ Normalized time factor

$D_{r i} \quad$ Hydrodynamic dispersion coefficient in the ith contaminated soil layer

$D_{m i} \quad$ Mechanical dispersion coefficient in the ith contaminated soil layer

$v_{i} \quad$ Seepage velocity of the $i$ th contaminated soil layer

$v_{a} \quad$ Darcy flow rate of the double-layered contaminated soil layer system

$K_{a} \quad$ Permeability coefficient of the double-layered contaminated soil layer system

\section{Acknowledgment}

This research is supported by National Natural Science Foundation of China (No. 52078467).

\section{Author Contributions}

X.L. (Ph.D.) derived the formulations and wrote the manuscript. J.S. (M.S. student) analyzed related literatures and programmed the code. Z.Z. (Ph.D.) reviewed and edited the manuscript. Z.W. (Ph.D.) reviewed and edited the manuscript.

\section{References}

1. Chen YM. A fundamental theory of environmental geotechnics and its application. Chin. J. Geotech. Eng. 2014;36:1-46.

2. Rajapriya R, Poonguzhali N, Murugaiyan V. Analyzing The Characteristics Of Landfill Leachate On Expansive Soil And Its Impact On Liner Materials Reinforced With Discrete Random Fibers. Surf. Rev. Lett. 2019;26:1-10.

3. Pu H, Fox PJ. Model for Coupled Large Strain Consolidation and Solute Transport in Layered Soils. Int. J. Geomech.
2016;16:04015064.

4. Banaei S, Javid AH, Hassani AH. Numerical simulation of groundwater contaminant transport in porous media. Int. J. Environ. Sci. Technol. 2021;18:151-162.

5. Saim O, Sinan MT, Nurtac O. Simultaneous dry-sorption of heavy metals by porous adsorbents during sludge composting. Environ. Eng. Res. 2020;25:258-265.

6. Tahmasebi P, Taheri M, Gharaie MHM. Heavy metal pollution associated with mining activity in the Kouh-e Zar region, NE Iran. Bull. Eng. Geol. Environ. 2020;79:1113-1123.

7. Chen YM, Xie KH, Ke H, et al. An analytical solution for one-dimensional contaminant diffusion through multi-layered system and its applications. Environ. Geol. 2009;58:1083-1094.

8. Johnson RL, Cherry JA, Pankow JF. Diffusion contaminant transport in natural clay: A field example and implications for clay-lined waste disposal sites. Environ. Sci. Technol. 1989;23: 340-349.

9. You X, Liu S, Dai C, et al. Contaminant occurrence and migration between high- and low-permeability zones in groundwater systems: A review. Sci. Tot. Environ. 2020;743:140703.

10. Yang Y, Wang M. Cation Diffusion in Compacted Clay: A Pore-Scale View. Environ Sci. Technol. 2019;53:1976-1984.

11. Pu HF, Qiu JW, Zhang R, et al. Analytical solutions for organic contaminant diffusion in triple-layer composite liner system considering the effect of degradation. Acta. Geotech. 2020;15: 907-921.

12. Thoma GJ, Reible DD, Valsaraj KT, Thibodeaux LJ. Efficiency of capping contaminated sediments in situ. 2. Mathematics of diffusion adsorption in the capping layer. Environ. Sci. Technol. 1993;27:2412-2419.

13. Malusis MA, Shackelford CD. Predicting solute flux through a clay membrane barrier. J. Geotech. Geoenviron. 2004;130: 477-487.

14. Foose GJ, Benson CH, Edil TB. Analytical equations for predicting concentration and mass flux from composite liners. Geosynth. Int. 2001;8:551-575.

15. Nima H, Paria P. Improving the permeability and adsorption of phenol by organophilic clay in clay liners. Environ. Eng. Res. 2020;25:96-103.

16. Tun MM, Juchelková D. Drying methods for municipal solid waste quality improvement in the developed and developing countries: A review. Environ. Eng. Res. 2019;24:529-542.

17. Jaume B. Soil contamination and human health: part 3. Environ. Geochem. Hlth. 2020;12:4065-4071.

18. Barati S, Shourijeh PT, Samani N, Asadi S. Stabilization of iron ore tailings with cement and bentonite: a case study on Golgohar mine. Bull. Eng. Geol. Environ. 2020;79:4151-4166.

19. Zairi M, Rouis M. Numerical and experimental simulation of pollutants migration in porous media. Bull. Eng. Geol. Environ. 2000;59:231-238.

20. He J, Gao ZK, Zhan GC. Transport of organic contaminants through a composite liner. Hydrog. Eng. Geol. 2009;1:124-127.

21. Xie HJ, Chen YM, Lou ZH. An analytical solution to contaminant transport through composite liners with geomembrane defects. Sci. China. Technol. Sc. 2010;40:486-495.

22. Xie HJ, Lou ZH, Chen YM, et al. An analytical solution to contaminant advection and dispersion through a GCL/AL liner 
system. Chinese. Sci. Bull. 2010;55:2148-2155.

23. Park E, Zhan HB. Analytical solutions of contaminant transport from finite one-, two-, and three-dimensional sources in a finite-thickness aquifer. J. Contam. Hydrol. 2001;53:41-61.

24. Rowe RK, Nadarajah P. An analytical method for predicting the velocity field beneath landfills. Can. Geotech. J. 1997;34: 264-282.

25. Leij FJ, Genuchten MTV. Approximate analytical solutions for solute transport in two-layer porous-media. Transport. Porous. Med. 1995;18:65-85.

26. Liu C, Ball WP. Analytical modeling of diffusion-limited contamination and decontamination in a two-layer porous medium. Adv. Water. Resour. 1998;21:297-313.

27. Liu C, Ball WP, Ellis JH. An analytical solution to the one-dimensional solute advection-dispersion equation in multi-layer porous media. Transport. Porous. Med. 1998;30:25-43.

28. Shackelford CD, Lee JM. Analyzing diffusion by analogy with consolidation. J. Geotech. Geoenviron. 2005;131:1345-1359.

29. Chen YM, Xie HJ, Ke H, et al. Analytical solution of contaminant diffusion through multilayered soils. Chinese. J. Geotech. Eng. 2006;4:521-524.

30. Xie HJ, Tang XW, Chen YM, et al. One dimensional model for contaminant diffusion through layered media. J. Zhejiang Univ-Sc. A. 2006;12:2191-2195.

31. Li YC, Cleall PJ. Analytical solutions for contaminant diffusion in double-layered porous media. J. Geotech. Geoenviron. 2010;136:1542-1554.

32. Rowe RK. Long-term performance of contaminant barrier systems (45th Rankin Lecture). Geotechnique 2005;55:631-677.

33. Young HM, Johnson B, Carson D, et al. Characterization of infiltration rates from landfills: supporting groundwater modelling efforts. Environ. Monit. Assess. 2004;96:283-311.

34. Ozisik MN. Heat conduction. 2nd ed. New York: John Wiley and sons; 1993. p.236-272

35. Xi YH, Ren J, Hu ZX. Determination of diffusion coefficient and distribution coefficient of pollutant ions in clay media. Chin. J. Geotech. Eng. 2006;3:122-127.

36. Xie HJ, Lou ZH, Chen YM, Jin AM, et al. Analytical solution of convection-dispersion of pollutants passing through GCL/AL impervious layer. China J. Sci. Bull. 2010;55:2153-2163.

37. Ray S, Mondal D, Reuveni S. Péclet number governs transition to acceleratory restart in drift-diffusion. J. Phys. A-Math. Theor. 2019;52:1-10. 